\title{
ResearchOnline@JCU
}

This is the Accepted Version of a paper published in the Journal of Strength and Conditioning Research:

Okuno, Nilo M., Pedro, Rafael E., Leicht, Anthony, Ramos, Solange de Paula, and Nakamura, Fabio Y. (2014) Cardiac Autonomic Recovery After a Single Session of Resistance Exercise With and Without Vascular Occlusion. Journal of Strength and Conditioning Research, 28 (4). pp. 1143-1150. http://dx.doi.org/10.1519/JSC.0000000000000245 
JSCR-08-3831, Revision 1

ORIGINAL ARTICLE

\section{CARDIAC AUTONOMIC RECOVERY AFTER A SINGLE SESSION OF RESISTANCE EXERCISE WITH AND WITHOUT VASCULAR OCCLUSION}

Running head: Cardiac autonomic after resistance exercise

Nilo M. Okuno1,2, Rafael E. Pedro2, Anthony S. Leicht3, Solange de Paula Ramos2,4,

\section{Fábio}

\section{Y. Nakamura2}

Department of Physical Education, State University of Ponta Grossa, Ponta Grossa, PR, Brazil

2 Department of Physical Education, State University of Londrina, Londrina, PR, Brazil

3 Institute of Sport and Exercise Science, James Cook University, Townsville, Queensland, Australia.

4Department of Histology, State University of Londrina, Londrina, PR, Brazil.

$(\bowtie)$ Address for correspondence: Nilo Massaru Okuno, Departamento de Educação Física, Universidade Estadual de Ponta Grossa, Av. Gen. Carlos Cavalcanti, 4748 - Uvaranas. CEP: 84030-900.

E-mail: nilookuno@yahoo.com.br; Phone +55 (42) 3220-3300 
JSCR-08-3831, Revision 1

\section{ABSTRACT}

The aim of this study was to investigate the heart rate variability (HRV) following resistance training with and without vascular occlusion. It was hypothesized that low intensity with vascular occlusion would elicit comparable post-exercise HRV responses to that of high intensity without vascular occlusion. Nine subjects undertook four experimental sessions of leg press exercise on different days: 1) one repetition maximum (1RM) test, 2) 4 sets of 8 repetitions + one set until exhaustion at $80 \%$ of $1 \mathrm{RM}$ without vascular occlusion (HI), 3) 4 sets of 16 repetitions + one set until exhaustion at $40 \%$ of $1 \mathrm{RM}$ with vascular occlusion (LIO), and 4 ) 4 sets of 16 repetitions + one set with the number of repetition equal to the last set of LIO but at $40 \%$ of 1RM without vascular occlusion (LI). HRV was analyzed 10min, 20min, 30min, $1 \mathrm{~h}$, $5 \mathrm{~h}$ and $24 \mathrm{~h}$ after HI, LIO and LI sessions. The HI session increased heart rate (HR) and reduced the root mean square of successive difference of R-R intervals (RMSSD) and log-transformed high frequency (lnHF) power during prolonged recovery $(\mathrm{HR}=5 \mathrm{~h} ; \mathrm{RMSSD}=30 \mathrm{~min} ; \mathrm{lnHF}=1 \mathrm{~h})$ at a greater magnitude when compared with LIO and LI. Despite the same intensity of exercise for LIO and LI, the occlusion delayed the recovery of $\mathrm{HR}$ and $\mathrm{HRV}$ variables. Post-exercise blood lactate concentration was moderate to strongly correlated with peak HR ( $r=0.87)$, RMSSD $(r=-0.64)$ and $\operatorname{lnHF}(\mathrm{r}=-0.68)$. The present study has demonstrated that LIO was able to reduce cardiac autonomic stress when compared with HI.

Keywords: Autonomic control, Kaatsu training, blood flow restriction, blood lactate concentration. 
JSCR-08-3831, Revision 1

\section{INTRODUCTION}

The American College of Sports Medicine (1) recommends resistance training to be performed with loads $>70 \%$ of one repetition maximum (1RM) in order to elicit increases in strength and muscle mass. However, high intensity resistance exercise may not be feasible for individuals recovering from orthopedic injuries, with some chronic diseases and for elderly people, all of whom may be unable to tolerate excessively stressful mechanical and metabolic changes. Recent evidence suggests that vascular occlusion training, so-called Kaatsu training, can increase strength and muscle mass to the same extent as traditional high-intensity resistance training while using substantially lower loads $(20-50 \%$ 1RM) $(8,21,34)$. The vascular occlusion during resistance training partially or totally obstructs the venous return, increasing the concentration of local metabolic factors (e.g. potassium and hydrogen ions) that stimulate the type III and IV afferent receptors and the mechanisms of hypertrophy, with higher anabolic hormones release and increase in muscle strength (23).

Kaatsu training has been applied in athletes, healthy persons and with different type of disease, obesity and elderly (27). This type of training has showed many benefits such as improving the sprint time and countermovement jump performance (9), increase bone mineral density (24), diminish disuse atrophy in individuals that underwent an operation for the reconstruction of the anterior cruciate ligament (33) and improving the functional capacity (11,37). Furthermore, some studies have shown that Kaatsu training is practical and safe for individuals with different cardiovascular diseases $(25,27)$. However, Iida et al. (13) showed that vascular occlusion (without exercise) induced an increase in sympathetic nervous system activity and a decrease of 
JSCR-08-3831, Revision 1

vagal activity, as analyzed by heart rate (HR) variability (HRV). Accordingly, Kluess and Wood (20) observed that vascular occlusion during handgrip exercise induced more pronounced vagal withdrawal concomitant with increased sympathetic activity when compared with the condition without vascular occlusion. These studies have demonstrated significant changes in cardiac autonomic modulation at rest and during exercise, yet the post-exercise cardiac autonomic response to Kaatsu training has not been described to date. Kaatsu training may have important physiological and clinical implications if cardiac autonomic control (e.g. HRV) is severely disturbed by exercise combined with occlusion. For example, impaired post-exercise parasympathetic reactivation has been reported to increase the risk of cardiac arrhythmias and sudden death $(2,35)$. In contrast, potential swift restoration of HRV following Kaatsu exercise may reduce the risk of future cardiovascular events as well as provide favorable physiological conditions for training adaptations in subsequent exercise (19).

Post-exercise parasympathetic reactivation is influenced by the exercise session loading (e.g. intensity and volume) and individual characteristics (e.g. age and physical fitness) $(16,31)$. Highintensity exercises involving large muscle mass and anaerobic metabolic stress are associated with delayed HRV recovery compared with lower intensity aerobic exercise $(5,28)$. However, with low intensity vascular occlusion training (LIO, $40 \%$ of one repetition maximum - 1RM), blood lactate concentration at the termination of exercise was comparable to that induced by high intensity (80\% of 1RM, (HI)), and higher than that for low intensity (LI, $40 \%$ of 1RM) without vascular occlusion (34). These results indicated that occlusive exercise (i.e. anaerobic stress) induced similar physiological stress to that of higher intensity exercise that may result in prolonged recovery. However, to our knowledge, HRV examination following occlusive 
JSCR-08-3831, Revision 1

resistance exercise has not been examined and may provide a greater understanding of the possible cardiac and exercise risks and physiological demands elicited by Kaatsu training. Thus, the purpose of this study was to investigate the cardiac autonomic stress (i.e. HRV) following an acute bout of resistance training with and without vascular occlusion. It was hypothesized that LIO would elicit comparable post-exercise HRV responses and cardiovascular stress due to the slow parasympathetic recovery and similar cardiovascular demands to that elicited by HI. This response will indicate if the traditional strength training and Kaatsu training may or may not be applied indistinctly for individuals with higher cardiovascular risk and the time-frame necessary to cardiac autonomic recovery after these types of exercises.

\section{METHODS}

\section{Experimental Approach to the Problem}

Kaatsu training induces muscle strength and hypertrophy in similar manner than conventional strength training. However, the cardiac autonomic stress induced by exercise is an important factor to be considered due the acute risks imposed by training and the time necessary to recovery for the subsequent session. Thus, subjects underwent four experimental sessions of unilateral leg press exercise analyzing the HRV recovery. The first session was used to determine the 1RM load. On the following three sessions they performed five sets for each lower limb of resistance exercise at: 1) 4 sets of 8 repetitions +1 set until exhaustion at high intensity exercise (80\% of 1RM) without vascular occlusion (HI); 2) 4 sets of 16 repetitions +1 set until exhaustion at low intensity exercise (40\% of 1RM) with vascular occlusion (LIO); and 3$) 4$ sets of 16 repetitions +1 set with same number of repetitions of LIO at low intensity exercise (40\% of 1RM) without vascular occlusion (LI). After the sessions, the HRV was recorded to verify the 
JSCR-08-3831, Revision 1

effects of intensity and vascular occlusion on cardiac autonomic modulation during recovery (10 $\min , 20 \mathrm{~min}, 30 \mathrm{~min}, 1 \mathrm{~h}, 5 \mathrm{~h}$ and $24 \mathrm{~h}$ ).

\section{Subjects}

Nine male participants between 20 and 29 years old $(24.0 \pm 2.9$ years; $174 \pm 7 \mathrm{~cm} ; 77.1 \pm 8.3 \mathrm{~kg})$ with at least 6 months of experience in resistance training took part within the present study. Participants attended the laboratory on four occasions. The first visit determined participants' maximal dynamic strength with the remaining three visits including the performance of resistance exercise sessions with or without vascular occlusion. Prior to the study, participants completed the physical activity readiness questionnaire (PAR-Q) with all confirmed as free of injuries and chronic diseases. Written informed consent was obtained from all participants after explanation about the aims, risks, and benefits involved in the study. During the study, participants were instructed to refrain from exhaustive exercise, avoid caffeine and alcohol ingestion for $24 \mathrm{~h}$ preceding and after the tests and to sleep for a minimum of $6 \mathrm{~h}$ in the night preceding the exercise session. Furthermore, they were also instructed to maintain the same diet habits throughout the study period. The study was approved by the local Ethics Committee and performed in accordance with the ethical standards of the Declaration of Helsinki.

\section{One Repetition Maximum Test (1RM)}

The participants attended one session at the University gym to determine maximal leg strength for the left and right legs via unilateral horizontal leg press exercise (Riguetto Fitness Equipment, Campinas, Brazil). Prior to maximal determination, participants ran on a treadmill (Embreex $563 \mathrm{R} 1$, Brusque, Brazil) at $8.5 \mathrm{~km} \cdot \mathrm{h}^{-1}$ for 5 minutes followed by five repetitions at 
JSCR-08-3831, Revision 1

approximately $50 \%$ of the estimated $1 \mathrm{RM}$, three repetitions at $70 \%$ of the estimated $1 \mathrm{RM}$ and one repetition at $90 \%$ of the estimated 1RM on the unilateral leg press. Subsequently, participants undertook five consecutive trials with each lower limb separately to determine the 1RM load with 3 minutes of rest between trials. The order of the single lower limb testing (i.e. left and right) was randomized. We opted to study the leg press exercise because it is a multiple joint exercise in which higher metabolic demand can be elicited than in single joint exercise as commonly recommended for resistance training by ACSM (1).

\section{Experimental Sessions}

Approximately three days after the $1 \mathrm{RM}$ assessment, the participants undertook three experimental sessions on different days, separated by at least $72 \mathrm{~h}$ and maximum of $120 \mathrm{~h}: 1$ ) five sets of leg press exercise at $80 \%$ of $1 \mathrm{RM}$ without vascular occlusion (HI), 2) five sets of leg press exercise at $40 \%$ of 1RM with vascular occlusion (LIO), and 3) five sets of leg press exercise at $40 \%$ of 1RM without vascular occlusion (LI) of each leg (Figure 1). The lower limbs were alternated during the execution of each set, and the first leg to be exercised by each participant was randomly assigned and maintained for all sessions. During the first 4 sets of HI and LIO sessions, participants performed 8 and 16 repetitions, respectively, while the fifth set consisted of continuous repetitions until exhaustion (i.e. inability to complete an additional successful lift). During the LI session, participants performed the same number of repetitions as in the LIO session. The order of HI and LIO sessions was randomized with the LI session always performed after both HI and LIO sessions to ensure a similar number of repetitions within the same low intensity exercise. The rest interval between each set was one minute (Figure 2). All sessions were conducted using an unilateral horizontal leg press machine (Riguetto Fitness 
JSCR-08-3831, Revision 1

Equipment, Campinas, Brazil) and performed in the morning (08:00-11:00 hrs) to minimize the circadian influences on muscular strength and cardiac autonomic responses (10). Peak HR during each session was recorded via a portable cardiac monitor (Polar RS800CX, Kempele, Finland).

\section{*** INSERT FIGURE 1 *** \\ *** INSERT FIGURE $2 * * *$}

During the LIO session, a standard blood pressure cuff (width $14 \mathrm{~cm}$; length $70 \mathrm{~cm}$ ) was applied around the thighs of both lower limbs and immediately distal to the inguinal fold. The cuff was inflated to $100 \mathrm{mmHg}$ and the pressure was maintained throughout the exercise session, including rest between sets (i.e. both legs were occluded during trials for each limb). This occlusion pressure has been previously reported to elicit $\sim 80 \%$ of total vascular occlusion (22).

\section{Blood Lactate Concentration}

Twenty five microliters of blood was collected from the earlobe at three minutes after the end of each session using heparinized capillary tubes (Figure 2). Blood samples were then subsequently placed in tubes containing $\mathrm{NaF}(1 \%)$ and stored on ice for $\sim 30$ minutes. The blood lactate concentration ([La]) was determined with an electrochemical device (YSI 1500 Select, Ohio, USA).

\section{Heart Rate Variability}

Five-minute, seated rest recordings of beat-to-beat (R-R) intervals were obtained before the exercise sessions and at 10 minute, 20 minute, 30 minute, 1 hour, 5 hours and 24 hours after each 
JSCR-08-3831, Revision 1

session with a portable cardiac monitor (Polar RS800CX, Kempele, Finland) and analyzed by Kubios HRV (version 2.0) software. Participants adhered to the 5-minutes stabilization period and all measurements were completed in the seated position and supervised by an experienced researcher. During first 30 minutes of recovery, participants remained seated on a chair in the gym for assessment of HRV. For the remaining measurements at 1 hour, 5 hours and 24 hours after each session, participants returned to the lab and undertook a minimum 10 min seated position for the recording of HRV (Figure 2). The data were analyzed in both the time and frequency domains with ectopic beats and artifacts $(<2 \%$ of recording) visually checked and corrected manually. The time domain variables examined included mean HR and the root mean square of successive difference of R-R intervals (RMSSD). The frequency domain indices were derived by a Fast Fourier Transform (256 s window with 50\% overlap) of the detrended tachogram of R-R intervals with the high frequency $(\mathrm{HF}-0.15-0.40 \mathrm{~Hz})$ and low frequency (LF - 0.04-0.15 Hz) expressed in absolute and normalized units, and as the LF/HF ratio. Buchheit et al. (7) presented good reliability (intraclass coefficient correlation $=0.69-0.88$ ) of most of these variables after exercise.

\section{Statistical Analyses}

The data are presented as mean \pm standard deviation. The Gaussian distribution was observed by Shapiro-Wilk test, with non-normal data log-transformed prior to statistical analysis. The right and left lower limb strength were compared by paired Student t-test. A one-way repeated measures ANOVA was used to compare the peak HR and [La] values among HI, LIO and LI sessions with a two-way (time $\mathrm{x}$ session) repeated measures ANOVA used to compare the HRV variables. Sphericity was analyzed by Mauchly's test followed by Greenhouse-Geisser 
JSCR-08-3831, Revision 1

correction, when necessary. Post-hoc analyses of ANOVA significances were conducted using the LSD post-hoc test. Relationships between [La] with peak HR and HRV variables were examined via Pearson or Spearman correlation coefficients, where appropriate. The level of significance was set at $\mathrm{P}<0.05$.

\section{RESULTS}

The participants exhibited a greater 1RM for the right compared with the left lower limb (129 \pm $21 \mathrm{~kg}$ vs. $121 \pm 22 \mathrm{~kg}, \mathrm{P}<0.05)$. Peak HR was significantly different for all sessions with higher HR during the HI session, compared to LIO which was greater than LI (165 \pm 16 bpm vs. $137 \pm$ 14 bpm vs. $112 \pm 10$ bpm, $\mathrm{P}<0.05)$. A similar result was observed for [La] with values for HI $(6.9 \pm 2.1 \mathrm{mM})$ significantly greater than LIO $(4.1 \pm 0.9 \mathrm{mM})$ which was significantly greater than $\mathrm{LI}(2.7 \pm 1.2 \mathrm{mM})(\mathrm{P}<0.05)$.

Following exercise, HR remained elevated for 30 minutes (LI and LIO) to 5 hours (HI) with HI values greater than LI at all-time points (Figure 3). Post-exercise HR for HI was greater than for LIO only during the first 30 minutes (Figure 3). In contrast, HR for LI fell below resting levels at 1-24 hours post-exercise which were also significantly different to LIO values (Figure 3).

\section{*** INSERT FIGURE 3 ***}

Post-exercise, RMSSD was reduced below resting values for 10 (LIO) and 30 minutes (HI) thereafter returning to resting levels (Figure 4). In contrast, RMSSD for LI remained similar to resting levels until 24 hours post-exercise when it significantly increased (Figure 4). Lower 
JSCR-08-3831, Revision 1

RMSSD values were observed for HI compared to LI for up to 1 hour post-exercise while values for LIO were significantly higher compared to $\mathrm{HI}$ at 10 and 30 minutes post-exercise (Fig. 4).

*** INSERT FIGURE $4 * * *$

For the frequency domain HRV indices, $\operatorname{lnLF}\left(\mathrm{ms}^{2}\right)$ was reduced from rest values at 10 minutes following $\mathrm{HI}$ and at 1 and 5 hours following LIO only (Table 1). During recovery, $\operatorname{lnLF}\left(\mathrm{ms}^{2}\right.$ ) values were significantly greater for LI and LIO compared to $\mathrm{HI}$ at 10 minutes post-exercise while values for LIO were significantly lower compared to LI at 1 and 5 hours post-exercise (Table 1). For $\operatorname{lnHF}\left(\mathrm{ms}^{2}\right)$, significantly lower values compared to rest were evident for up to 1 hour following HI and LIO exercise with the HI values significantly lower than LIO and LI values for the first 30 minutes of recovery (Table 1). At the later stages of recovery (5-24 hours), $\operatorname{lnHF}\left(\mathrm{ms}^{2}\right)$ was significantly greater for LI compared with LIO (Table 1). Post-exercise, all other HRV indices (nu and LF/HF) were similar to resting levels and similar between conditions (i.e. HI, LI and LIO, Table 1).

*** INSERT TABLE $1 * * *$

The post-exercise [La] was moderate to strongly correlated with peak HR and parasympathetic HRV indices at 10 minutes post-exercise (Figure 5). Weaker correlations were also identified between post-exercise [La] and $\operatorname{lnLF}\left(\mathrm{ms}^{2}\right), \mathrm{LF}(\mathrm{nu}), \mathrm{HF}(\mathrm{nu})$, and LF/HF (Figure 5).

*** INSERT FIGURE $5 * * *$

\section{DISCUSSION}


JSCR-08-3831, Revision 1

The aim of this study was to investigate the cardiac autonomic stress following an acute bout of resistance training with and without vascular occlusion. Different to our initial hypothesis, the current study has demonstrated that HI exercise resulted in a significant increase in post-exercise HR accompanied by a significant reduction of HRV that is greater than that observed following LI and LIO exercise. Further, the change in HR and HRV post-exercise was greater for LIO compared with LI indicating a greater cardiac autonomic disturbance with occlusion. These results and significant correlations between $[\mathrm{La}]$ and post-exercise HRV indicate that exercise intensity and occlusion alters cardiac autonomic control for some time post-exercise, possibly via changes in local metabolites.

Immediately after exercise, HR recovers in two distinct phases, a fast decrease due to the increase of parasympathetic activity and a slower reduction due to the withdrawal of sympathetic activity $(4,15)$. During recovery after HI, HRV was observed to be delayed over time suggesting a blunted parasympathetic recovery (i.e. RMSSD and HF) compared with the LIO and LI protocols. Differences between protocols may reflect the known impact of exercise intensity on recovery $(17,26)$. Interestingly, the recovery following LIO was delayed when compared with LI and indicates a greater autonomic disruption when blood flow is occluded during exercise. The same load and number of repetitions was conducted during LIO and LI and therefore differences most likely indicate occlusion as a contributing factor to post-exercise cardiac autonomic control rather than exercise intensity. Similar reductions in HRV have been noted during rest during thigh occlusion of 45-200 mmHg (14) indicating a direct influence of restricted blood flow on cardiac autonomic modulations. 
JSCR-08-3831, Revision 1

Potentially, the different HRV recoveries may be influenced by the anaerobic contribution of exercise as measured by [La]. Buchheit et al. (5) reported that an all-out sprint exercise induced higher blood lactate accumulation $(10.9 \pm 0.9 \mathrm{mM})$ and impaired parasympathetic reactivation when compared with moderate, isocaloric, continuous exercise $(3.5 \pm 0.2 \mathrm{mM})$. Our results are in accordance with this evidence as higher [La] may have delayed HRV recovery after HI session compared to LIO and LI due to the accumulation of metabolic by-products (5). Furthermore, the [La] concentration was inversely correlated with parasympathetic indices $(\mathrm{RMSSD}=-0.64 ; \ln H \mathrm{~F}$ $=-0.68)$. The elevated metabolic demand during exercise may have increased muscle engagement and adrenergic activity that consequently delayed HRV recovery when compared with the low intensity exercise. Further, exercise with occlusion (LIO) most likely resulted in a greater muscle activation compared with its equivalent non occluded exercise as prior work has reported significant correlations between [La] and muscle activity following exercise with and without occlusion (36). However, the current results are different to that observed by Takarada et al. (34) in which [La] at the end of exhaustive LIO exercise was similar to that of HI. This difference could be due to the different muscle masses utilized with Takarada et al. (34) incorporating a smaller mass (elbow flexion) than the current study (leg press). The present results therefore indicated that LIO exercise results in lower metabolic (i.e. [La]) and cardiac stress (e.g. HR) during exercise when compared with traditional HI resistance training. Given that LIO exercise results in similar muscular hypertrophy and strength gains to that of HI exercise $(18,21,32)$, LIO exercise may provide the elderly and patients with osteomyoarticular and cardiovascular diseases a safer and beneficial form of training with less metabolites and less potential risk of lethal cardiac arrhythmias and sudden death (2). 
JSCR-08-3831, Revision 1

While LIO exercise resulted in a delayed HRV recovery compared to similar LI exercise, an interesting feature of LI exercise was an increase of parasympathetic activity (Figure 4) and concomitant reduction of HR (Figure 3), 24 hours post-exercise. This HRV increase at a given moment after exercise has been referred to as vagal rebound (12,30). Hautala et al. (12) observed an increase in HF, associated with a reduced LF and LF/HF two days after a $75 \mathrm{~km}$ cross-country skiing race. Likewise, Pober et al. (30) reported that HRV increased 1 and 22 hours following 60 minutes of cycling exercise at $65 \%$ of $\mathrm{VO}_{2} \mathrm{max}$ in comparison to the control (rest) situation. The mechanisms underlying vagal rebound are not totally clear; however, some authors have speculated that the exercise induced plasma volume increase activates baroreflex mechanisms leading to a delayed overexpression of parasympathetic activity (6). In the current study, vagal rebound occurred only following LI exercise with a longer recovery time (e.g. $\geq 48 \mathrm{~h}$ ) possibly needed to observe similar rebounds and full parasympathetic recovery after the HI and LIO sessions.

The HRV response presented in the our study indicate that the traditional strength training and Kaatsu training may not be applied indistinctly for individuals with higher cardiovascular risk and the time-frame necessary to cardiac autonomic recovery after Kaatsu training in lower than traditional strength training. It is important to prescribe the exercise in some populations and the subsequent session of training considering the time necessary to cardiovascular recovery.

A limitation of the present study was not controlling the breathing frequency due to the possible discomfort associated with this maneuver after exercise. Breathing frequency has been shown to affect thoracic stretch receptor afferents which can produce a reflex inhibition of vagal motor 
JSCR-08-3831, Revision 1

outflow and consequently influence HRV spectral components $(3,29)$. However, controlling for breathing frequency in the post-exercise period has been reported to produce acceptable and reliable results (7) with future studies necessary to examine potential breathing influences on post-exercise HRV.

\section{PRACTICAL APPLICATIONS}

Kaatsu training has been applied in different populations (i.e. athletes, healthy persons, person with different type of disease, obesity and elderly) and showed many benefits such as improve performance, increase bone mineral density, diminish disuse atrophy and improving the functional capacity. The current study has demonstrated that Kaatsu resistance training may provide favorable physiological state (i.e. lower metabolic stress and post-exercise disturbance of cardiac autonomic modulation) for training-induced benefits (strength and muscle mass increase) with lighter loads $(20-50 \%$ of $1 \mathrm{RM})$ than traditional strength training (>70\% of 1RM). The HRV recovery was longer and delayed with increasing resistance exercise intensity, irrespective of vascular occlusion, and the occlusion during exercise induced a distinct recovery time course when compared with the same intensity and number of repetitions of single leg press performed without occlusion. Delayed parasympathetic and HRV recovery following occlusive, resistance exercise may result in part from the higher [La]. It can be clinically by potentially lowering the risk of cardiac complication when compared to high intensity exercise in selected individuals who are advised to avoid performing high intensity exercise. 
JSCR-08-3831, Revision 1

\section{REFERENCES}

1. American College of Sports Medicine Position Stand. Progression models in resistance training for healthy adults. Med Sci Sports Exerc 41: 687-708, 2009.

2. Billman, GE. Cardiac autonomic neural remodeling and susceptibility to sudden cardiac death: effect of endurance exercise training. Am J Physiol Heart Circ Physiol 297: H1171-H1193, 2009.

3. Bloomfield, DM, Magnano, A, Bigger JR, JT, Rivadeneira, H, Parides, M, Steinman, RC. Comparison of spontaneous vs. Metronome-guided breathing on assessment of vagal modulation using RR variability. Am J Physiol Heart Circ Physiol 280: H1145-H1150, 2001.

4. Borresen, J, Lambert, MI. Autonomic control of heart rate during and after exercise: measurements and implications for monitoring training status. Sports Med 38: 633-646, 2008.

5. Buchheit, M, Laursen, PB, Ahmaidi, S. Parasympathetic reactivation after repeated sprint exercise. Am J Physiol Heart Circ Physiol 293: H133-H141, 2007.

6. Buchheit, M, Laursen, PB, Al Haddad, H, Ahmaidi, S. Exercise-induced plasma volume expansion and post-exercise parasympathetic reactivation. Eur J Appl Physiol. 105: 471-481, 2009.

7. Buchheit, M, Millet, GP, Parisy, A, Pourchez, S, Laursen, PB, Ahmaidi, S. Supramaximal training and postexercise parasympathetic reactivation in adolescents. Med Sci Sports Exerc 40: $362-371,2008$.

8. Clark, BC, Manini, TM, Hoffman, RL, Williams, PS, Guiler, MK, Knutson, MJ, McGlynn, ML, Kushnick, MR. Relative safety of 4 weeks of blood flow-restricted resistance exercise in young, healthy adults. Scand J Med Sci Sports 21: 653-662, 2011. 
JSCR-08-3831, Revision 1

9. Cook, CJ, Kilduff, LP, Beaven, CM. Three weeks of occlusion training can improve strength and power in trained athletes. Int J Sports Physiol Perform. In press.

10. Fürholz, M, Radtke, T, Roten, L, Tanner, H, Wilhelm, I, Schmid, JP, Saner, H, Wilhelm, M. Training-related modulations of the autonomic nervous system in endurance athletes: is female gender cardioprotective? Eur J Appl Physiol 113: 631-640, 2013.

11. Gualano, B, Neves, M Jr, Lima, FR, Pinto, AL, Laurentino, G, Borges, C, Baptista, L, Artioli, GG, Aoki, MS, Moriscot, A, Lancha, AH Jr, Bonfá, E, Ugrinowitsch, C. Resistance training with vascular occlusion in inclusion body myositis: a case study, Med Sci Sports Exerc 42: $250-254,2010$.

12. Hautala, A, Tulppo, MP, Mäkikallio, TH, Laukkanen, R, Nissilä, S, Huikuri, HV. Changes in cardiac autonomic regulation after prolonged maximal exercise. Clin Physiol 21: 238-245, 2001.

13. Iida, H, Kurano, M, Takano, H, Kubota, N, Morita, T, Meguro, K, Sato, Y, Abe, T, Yamazaki, Y, Uno, K, Takenaka, K, Hirose, K, Nakajima, T. Hemodynamic and neurohumoral responses to the restriction of femoral blood flow by KAATSU in healthy subjects. Eur J Appl Physiol 100: 275-285, 2007.

14. Iida, H, Takano, H, Meguro, K, Asada, K, Oonuma, H, Morita, T, Kurano, M, Sakagami, F, Uno, K, Hirose, K, Nagata, T, Takenaka, K, Suzuki, J, Hirata, Y, Furuichi, T, Eto, F, Nagai, R, Sato, Y, Nakajima, T. Hemodynamic and autonomic nervous responses to the restriction of femoral blood flow by KAATSU. Int J KAATSU Training Res 1: 57-64, 2005.

15. Imai, K, Sato, H, Hori, M, Kusuoka, H, Ozaki, H, Yokoyama, H, Takeda, H, Inoue, M, Kamada, T. Vagally mediated heart rate recovery after exercise is accelerated in athletes but blunted in patients with chronic heart failure. J Am Coll Cardiol 24: 1529-1535, 1994. 
JSCR-08-3831, Revision 1

16. Kaikkonen, P, Nummela, ., Rusko, H. Heart rate variability dynamics during early recovery after different endurance exercises. Eur J Appl Physiol 102: 79-86, 2007.

17. Kaikkonen, P, Rusko, H, Martinmäki, K. Post-exercise heart rate variability of endurance athletes after different high-intensity interventions Scand J Med Sci Sports 18, 511-519, 2008.

18. Karabulut, M, Abe, T, Sato, Y, Bemben, MG. The effects of low-intensity resistance training with vascular restriction on leg muscle strength in older men. Eur J Appl Physiol 108: 147- 155, 2010.

19. Kiviniemi, AM, Hautala, AJ, Kinnunen, H, Tulppo, MP. Endurance training guided individually by daily heart rate variability measurements. Eur J Appl Physiol 101: 743-751, 2007.

20. Kluess, HA, Wood, RH. Heart rate variability and exercise pressor reflex during dynamic handgrip exercise and postexercise arterial occlusion. Am J Med Sci 329: 117-123, 2005.

21. Kubo, K, Komuro, T, Ishiguro, N, Tsunoda, N, Sato, Y, Ishii, N, Kanehisa, H, Fukunaga, T. Effects of low-load resistance training with vascular occlusion on the mechanical properties of muscle and tendon. J Appl Biomech 22: 112-119, 2006.

22. Laurentino, G, Ugrinowitsch, C, Aihara, AY, Fernandes, AR, Parcell, AC, Ricard, M, Tricoli, V. Effects of strength training and vascular occlusion. Int J Sports Med 29: 664-667, 2008.

23. Loenneke, JP, Wilson, GJ, Wilson, JM. A mechanistic approach to blood flow occlusion. Int J Sports Med 31: 1-4, 2010.

24. Loenneke, JP, Young, KC, Fahs, CA, Rossow, LM, Bemben, DA, Bemben, MG. Blood flow restriction: rationale for improving bone. Med Hypotheses 78: 523-527, 2012. 
JSCR-08-3831, Revision 1

25. Madarame, H, Kurano, M, Fukumura, K, Fukuda, T, Nakajima, T. Haemostatic and inflammatory responses to blood flow-restricted exercise in patients with ischaemic heart disease. Clin Physiol Funct Imaging 33: 11-17, 2013.

26. Martinmäki, K, Rusko, H. Time-frequency analysis of heart rate variability during immediate recovery from low and high intensity exercise. Eur J Appl Physiol 102: 353-360, 2008.

27. Nakajima, T, Kurano, M, Iida, H, Takano, H, Oonuma, H, Morita, T, Meguro, K, Sato, Y, Nagata, T. Use and safety of KAATSU training: results of a national survey. Int $J$ KAATSU Training Res 2: 5-13, 2006.

28. Oliveira, RS, Vitor da Costa, M, Pedro, RE, Polito, MD, Avelar, A, Cyrino, ES, Nakamura, FY. Acute cardiac autonomic responses after a bout of resistance exercise. Sci Sports 27: 357$364,2012$.

29. Penttilä, J, Helminen, A, Jartti, T, Kuusela, T, Huikuri, HV, Tulppo, MP, Coffeng, R, Scheinin, H. Time domain, geometrical and frequency domain analysis of cardiac vagal outflow: effects of various respiratory patterns. Clin Physiol 21: 365-76, 2001.

30. Pober, DM, Braun, B, Freedson, PS. Effects of a single bout of exercise on resting heart rate variability. Med Sci Sports Exerc 36: 1140-1148, 2004.

31. Seiler, S, Haugen, O, Kuffel, E. Autonomic recovery after exercise in trained athletes: intensity and duration effects. Med Sci Sports Exerc 39: 1366-1373, 2007.

32. Takarada, Y, Sato, Y, Ishii, N. Effects of resistance exercise combined with moderate vascular occlusion on muscle function in athletes. Eur J Appl Physiol 86: 308-314, 2002.

33. Takarada, Y, Takazawa, H, Ishii, N. Applications of vascular occlusion diminish disuse atrophy of knee extensor muscles. Med Sci Sports Exerc 32: 2035-2039, 2000. 
JSCR-08-3831, Revision 1

34. Takarada, Y, Takazawa, H, Sato, Y, Takebayashi, S, Tanaka, Y, Ishii, N. Effects of resistance exercise combined with moderate vascular occlusion on muscular function in humans. J Appl Physiol 88: 2097- 2106, 2000.

35. Thompson, PD, Franklin, BA, Balady, GJ, Blair, SN, Corrado, D, Estes, NA, Fulton, JE, Gordon, NF, Haskell, WL, Link, MS, Maron, BJ, Mittleman, MA, Pelliccia, A, Wenger, NK, Willich, SN, Costa, F, American Heart Association Council on Nutrition, Physical Activity, and Metabolism; American Heart Association Council on Clinical Cardiology; American College of Sports Medicine. Exercise and acute cardiovascular events placing the risks into perspective: a scientific statement from the American Heart Association Council on Nutrition, Physical Activity, and Metabolism and the Council on Clinical Cardiology. Circulation 115: 2358-2368, 2007.

36. Yasuda, T, Fukumura, K, Fukuda, T, lida, H, Imuta, H, Sato, Y, Yamasoba, T, Nakajima, T. Effects of low-intensity, elastic band resistance exercise combined with blood flow restriction on muscle activation. Scand J Med Sci Sports. In press

37. Yokokawa, Y, Hongo, M, Urayama, H, Nishimura, T, Kai, I. Effects of low-intensity resistance exercise with vascular occlusion on physical function in healthy elderly people. Biosci Trends 2: 117-23, 2008. 
JSCR-08-3831, Revision 1

\section{ACKNOWLEDGMENTS}

The authors would like to thank Diego Costa Narciso for the assistance during data collection.

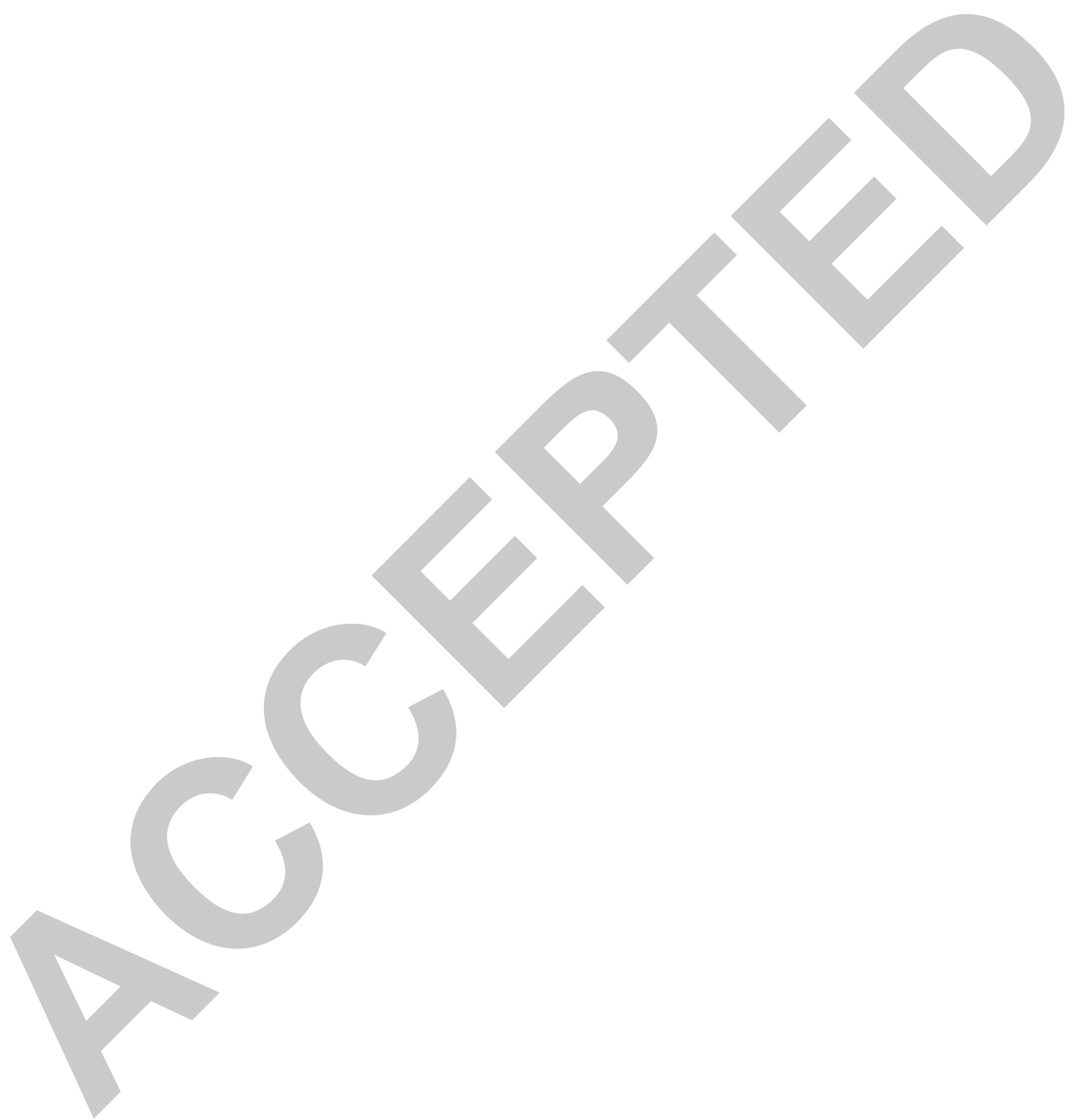


JSCR-08-3831, Revision 1

\section{FIGURE LEGENDS}

Figure 1. Schematic representation of the experimental sessions.

Figure 2. Schematic representation of the each experimental session.

Figure 3. Mean and standard deviation of heart rate during rest and at $10 \mathrm{~min}, 20 \mathrm{~min}, 30 \mathrm{~min}, 1$ $\mathrm{h}, 5 \mathrm{~h}$ and $24 \mathrm{~h}$ of recovery following high intensity $(\mathrm{HI})$, low intensity with vascular occlusion (LIO) and low intensity (LI) resistance exercise.

* Significantly different to rest $(\mathrm{P}<0.05)$;

\# Significantly different to HI at the same time $(\mathrm{P}<0.05)$;

+ Significantly different to LIO at the same time $(\mathrm{P}<0.05)$.

Figure 4. Mean and standard deviation of RMSSD during rest and at $10 \mathrm{~min}, 20 \mathrm{~min}, 30 \mathrm{~min}, 1$ $\mathrm{h}, 5 \mathrm{~h}$ and $24 \mathrm{~h}$ of recovery following high intensity (HI), low intensity with vascular occlusion (LIO) and low intensity (LI) resistance exercise.

* Significantly different to rest $(\mathrm{P}<0.05)$;

\# Significantly different to $\mathrm{HI}$ at the same time $(\mathrm{P}<0.05)$;

+ Significantly different to LIO at the same time $(\mathrm{P}<0.05)$.

Figure 5. Relationship between [La] with peak HR and HRV indices at 10 minutes post-exercise (RMSSD, $\operatorname{lnHF}\left(\mathrm{ms}^{2}\right), \operatorname{lnLF}\left(\mathrm{ms}^{2}\right), \mathrm{LF}(\mathrm{nu}), \mathrm{HF}(\mathrm{nu})$ and LF/HF). 
JSCR-08-3831, Revision 1

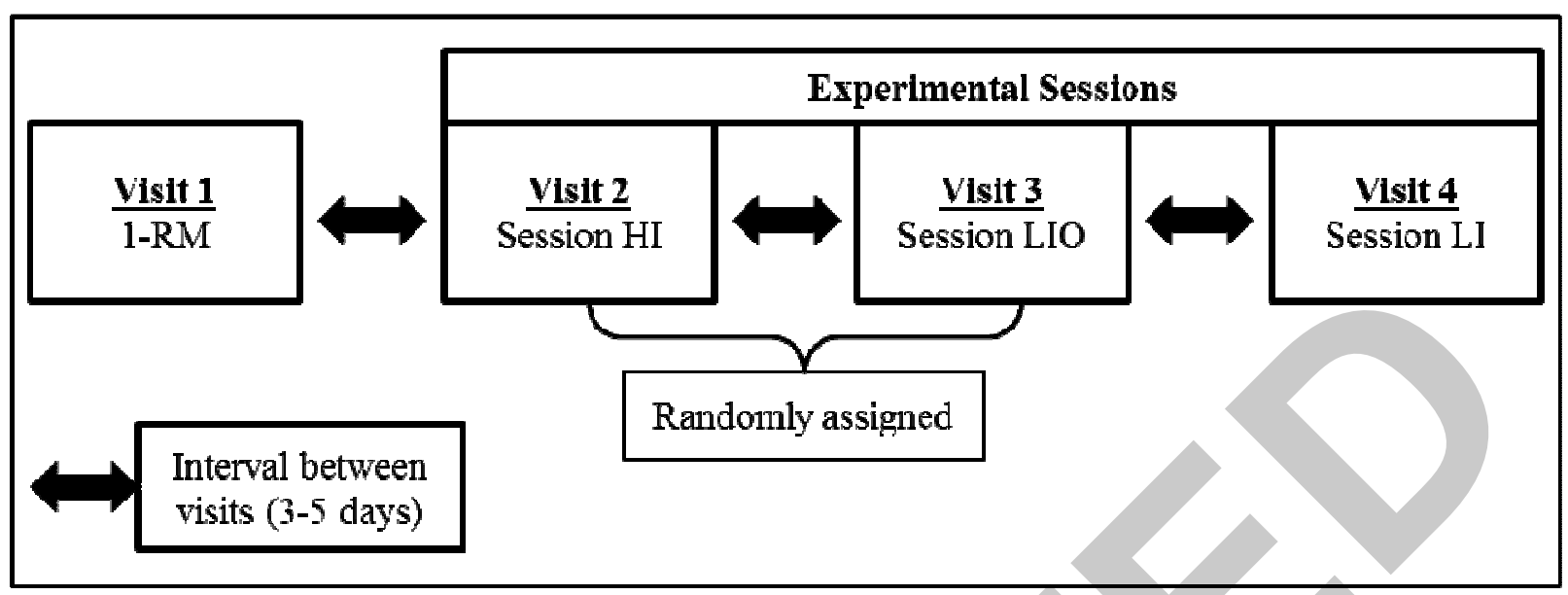

Figure 1. 
JSCR-08-3831, Revision 1

\begin{tabular}{|l|l|l|l|}
\hline & Rest & Experimental Sessions & Recovery \\
\hline 0 & HRV & $\mathbf{1 0}$ min & $\begin{array}{c}\text { During all session } \\
\text { HR assessment }\end{array}$ \\
\hline
\end{tabular}

\section{Figure 2.}


JSCR-08-3831, Revision 1

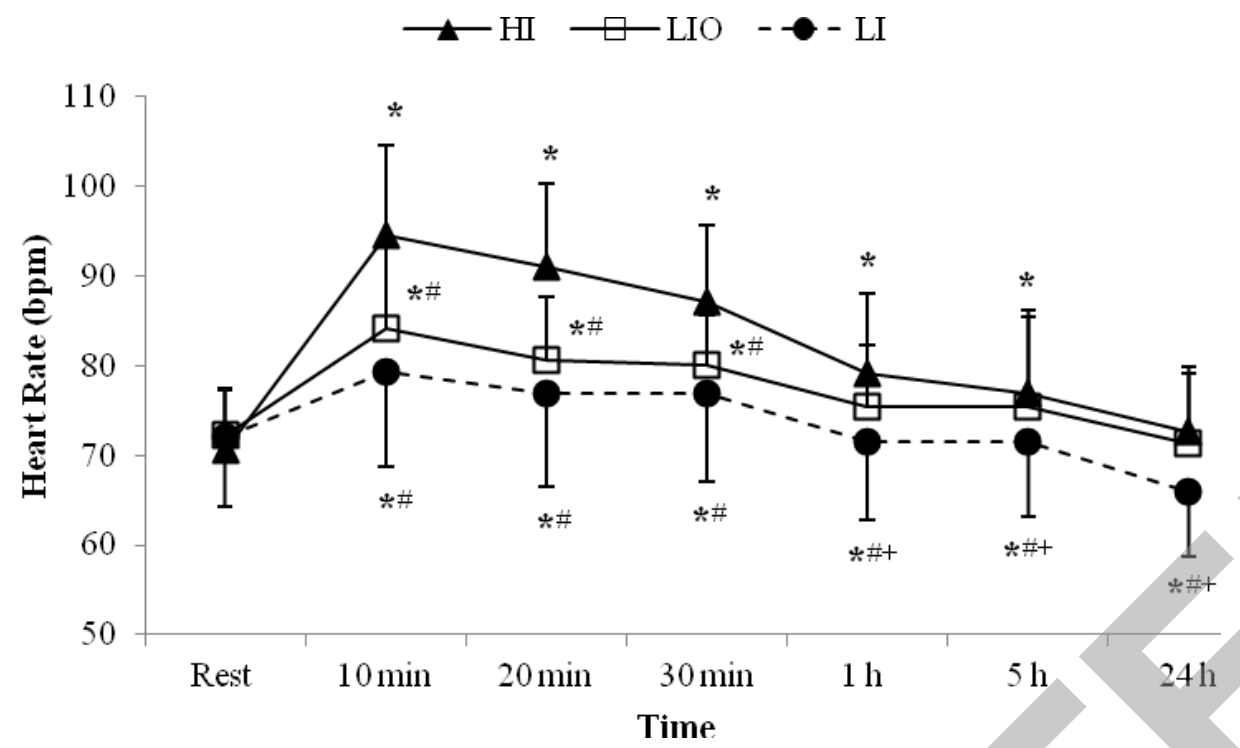

Figure 3. 
JSCR-08-3831, Revision 1
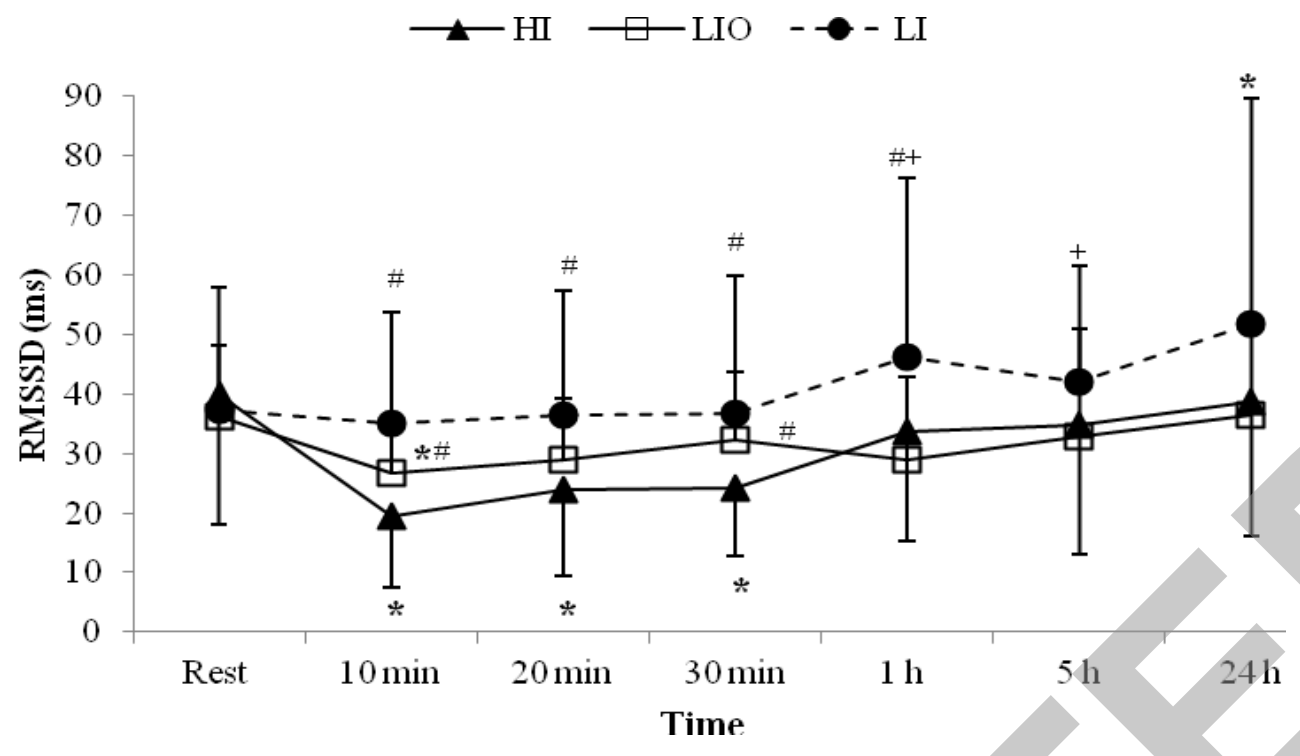

Figure 4. 
JSCR-08-3831, Revision 1
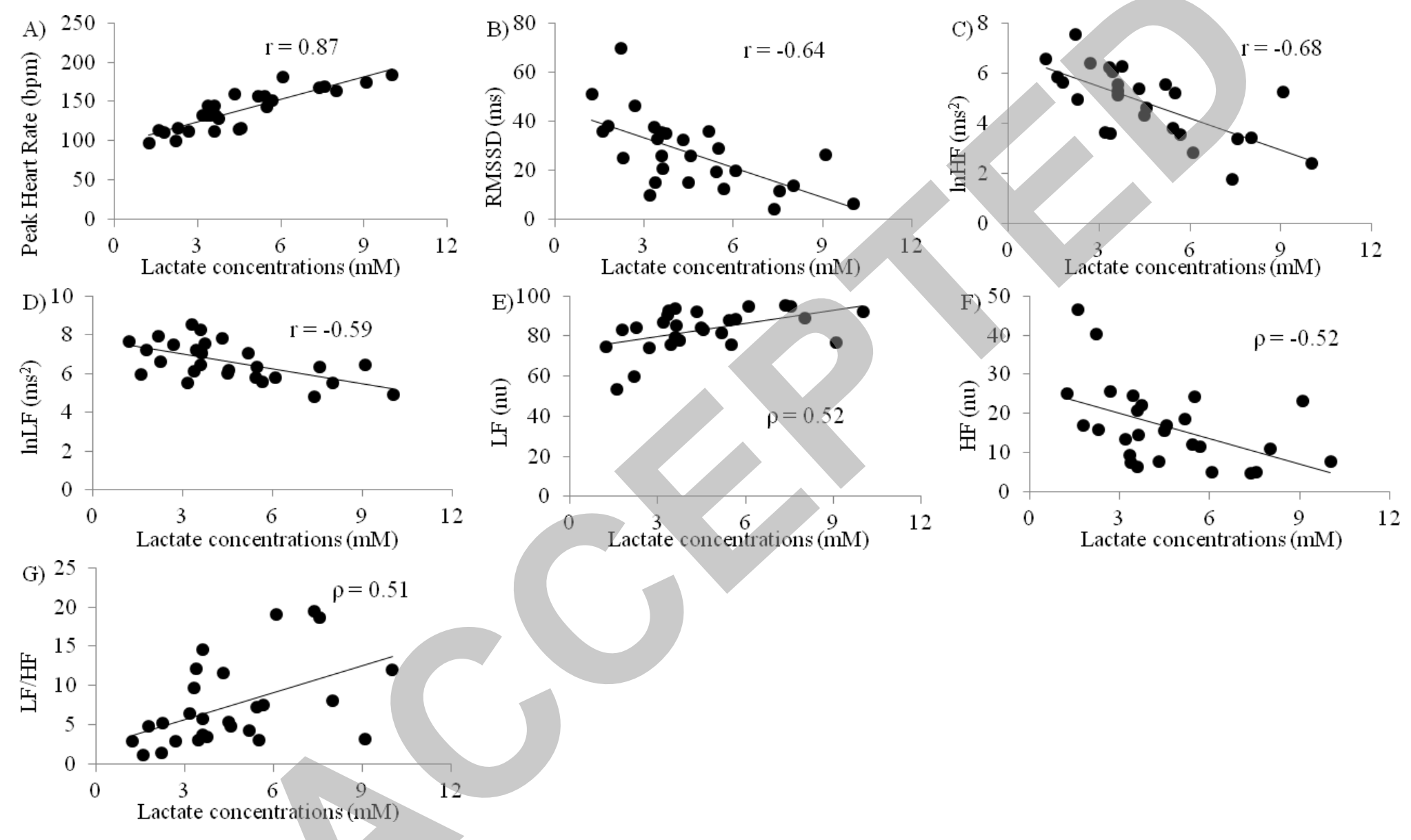

Figure 5. 
JSCR-08-3831, Revision 1

Table 1 - Mean \pm standard deviation of frequency domain indices of heart rate variability at rest and $10 \mathrm{~min}, 20 \mathrm{~min}, 30 \mathrm{~min}, 1 \mathrm{~h}, 5 \mathrm{~h}$ and $24 \mathrm{~h}$ of recovery following high intensity (HI), low intensity with vascular occlusion (LIO) and low intensity (LI) resistance exercise.

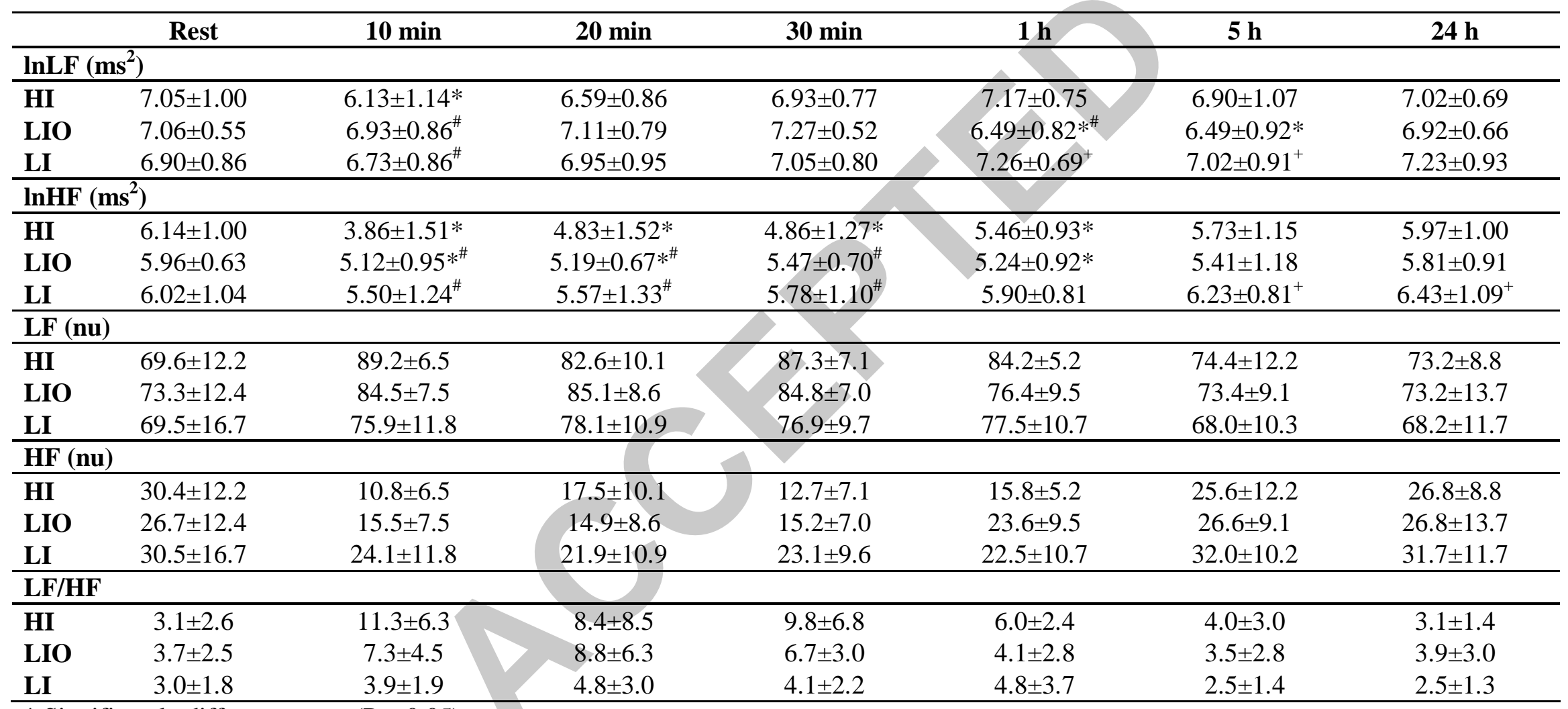

* Significantly different to rest $(\mathrm{P}<0.05)$;

\# Significantly different to $\mathrm{HI}$ at the same time $(\mathrm{P}<0.05)$;

${ }^{+}$Significantly different to LIO at the same time $(\mathrm{P}<0.05)$. 